\title{
Extraction of the Plane of Minimal Cross-Sectional Area of the Corpus Callosum Using Template-Driven Segmentation
}

\author{
Neda Changizi ${ }^{1}$, Ghassan Hamarneh ${ }^{1}$, Omer Ishaq ${ }^{1,2}$, \\ Aaron Ward ${ }^{1,3}$, and Roger Tam ${ }^{4}$ \\ ${ }^{1}$ Medical Image Analysis Lab, Simon Fraser University, Canada \\ ${ }^{2}$ Department of Computer Sciences, Air University, Pakistan \\ ${ }^{3}$ Robarts Research Institute, The University of Western Ontario, Canada \\ ${ }^{4}$ MS/MRI Research Group, University of British Columbia, Canada
}

\begin{abstract}
Changes in corpus callosum (CC) size are typically quantified in clinical studies by measuring the CC cross-sectional area on a midsagittal plane. We propose an alternative measurement plane based on the role of the $\mathrm{CC}$ as a bottleneck structure in determining the rate of interhemispheric neural transmission. We designate this plane as the Minimum Corpus Callosum Area Plane (MCCAP), which captures the cross section of the $\mathrm{CC}$ that best represents an upper bound on interhemispheric transmission. Our MCCAP extraction method uses a nested optimization framework, segmenting the $\mathrm{CC}$ as it appears on each candidate plane, using registration-based segmentation. We demonstrate the robust convergence and high accuracy of our method for magnetic resonance images and present preliminary clinical results showing higher sensitivity to disease-induced atrophy.
\end{abstract}

\section{Introduction}

The corpus callosum (CC) is an anatomic structure that acts as a communication bridge connecting the two brain hemispheres [1]. Certain neurological diseases are known to affect the shape and size of the CC. In particular, there have been numerous studies correlating CC measurements to multiple sclerosis [2], schizophrenia [3], autism [4, and many other mental and physical ailments. The accurate measurement of $\mathrm{CC}$ area changes is dependent on the repeatable identification of the cross-sectional plane of interest in all studied images. In previous studies, changes in CC size have been quantified by measuring its sagittal crosssectional area on a midsagittal plane (MSP).

MSP identification approaches can be classified as either symmetry- or featurebased. Symmetry-based approaches assume bilaterally symmetric hemispheres, with the MSP chosen to maximize this symmetry. Published approaches suggest different symmetry criteria; e.g., intensity ratios [5], cross-correlation [6], or edge-based [7. In feature-based approaches, the MSP is defined as the plane best matching the cerebral interhemispheric fissure. The Hough transform [ 8 ,

T. Jiang et al. (Eds.): MICCAI 2010, Part III, LNCS 6363, pp. 17-24, 2010.
(C) Springer-Verlag Berlin Heidelberg 2010 
orthogonal regression of B-spline snakes [9], and minimization of white matter area (including the cerebellum) 10, are examples of the feature-based approach.

It has been shown that even small errors in the MSP selection confound the interpretation of actual CC area changes due to pathology [11,12. Therefore, it may be clinically more meaningful to define the plane for CC atrophy measurement according to the characteristics of the $\mathrm{CC}$ itself rather than more global attributes such as brain symmetry or the interhemispheric fissure.

It is known that the cross-sectional area of the CC is proportional to the number of nerve fibers passing through it [1], and correlates with neural transmission [13, 14, 15, 16. Therefore, in this paper, we propose adopting the minimum $\mathrm{CC}$ cross-sectional area as the primary factor in defining the bottleneck (upper bound) of interhemispheric communication and hypothesize that the minimum $\mathrm{CC}$ cross-sectional area is a potentially more representative measure of $\mathrm{CC}$ degeneration as compared to areas of other cross sections.

We emphasize that we are proposing a new plane for the measurement of CC atrophy, and not a new criterion for MSP extraction. We refer to this plane as the Minimum CC Area Plane (MCCAP). We note that multiple planes in a given brain may have the same minimum CC area. However, since we postulate that all of these planes restrict the neural transmission similarly, the identification of any one of these planes results in satisfying our objectives for measuring the CC's structural health. We also note that the rate of $\mathrm{CC}$ degeneration may not be uniform for the whole $\mathrm{CC}$ and hence it is possible that the parameters defining the MCCAP may vary over time for the same patient.

\section{Method}

\subsection{Overview and Definitions}

We extract the MCCAP from a brain magnetic resonance imaging (MRI) volume by first defining an objective function that measures the cross-sectional area $A_{c c}$ of the $\mathrm{CC}$ in a given extracted plane. Then, using an iterative optimization procedure, $A_{c c}$ is minimized with respect to the set of plane parameters $P_{\text {ext }}$ (Fig. 1). Using an ASL coordinate system, the parameters of $P_{\text {ext }}$ are the angles $R_{x}$ and $R_{y}$ (in degrees) between the plane normal vector and the grid axes in the anterior and superior directions, respectively, and the distance $T_{z}(\mathrm{~mm})$ of the plane from the origin. The $\mathrm{CC}$ is segmented on every candidate plane by deformably registering a pre-segmented 2D template image from the same MRI volume to the extracted slice on the candidate plane. An alternative approach that one might suggest is to pre-segment the whole CC-bridge in 3D and find the plane with minimum $\mathrm{CC}$ area within the segmented volume. However, by using registration-based segmentation we avoid the complications of a full 3D segmentation, which is difficult to validate, even for anatomical experts.

\subsection{Template Preparation}

To create the template for the deformable registration step (Sec. 2.3), we extract the central sagittal slice from the MRI volume and provide an expert-segmented 


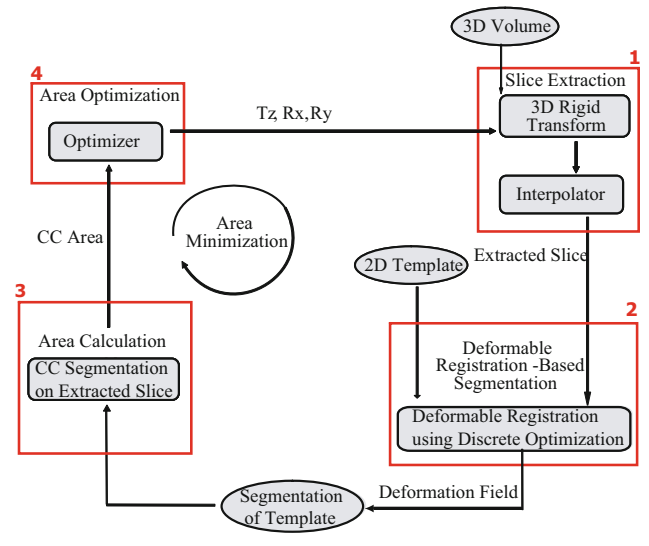

Fig. 1. Flow diagram of our method for finding the MCCAP. There are 4 primary steps. 1) A $2 \mathrm{D}$ slice is extracted (upper right) from the volume using $P_{\text {ext }}$. 2) The presegmented 2D template is registered (lower right) to the extracted slice through deformable registration. 3) The displacement field from the registration step is applied to the segmentation of the $2 \mathrm{D}$ template to segment the $\mathrm{CC}$ on the extracted slice. 4) The CC area and its derivatives with respect to $P_{\text {ext }}$ are used (upper left) for updating the values of $P_{\text {ext }}$ for the next iteration. Steps 1-4 are repeated until convergence.

$\mathrm{CC}$ on this slice. This central slice is positioned, either during acquisition or with post-processing, to align with the MSP. We hypothesize that most MSPs are spatially not very far from the MCCAP because the CC narrows from both sides toward the center of the brain and hence only a small spatial transformation is needed to reach the MCCAP from the MSP. In addition, we employ a multilevel optimization (Sec. 2.5) to further reduce the chances of converging onto a local minimum between the MSP and the MCCAP.

\subsection{Deformable Registration-Based Segmentation}

We nonlinearly register the presegmented 2D template to the extracted slice and apply the deformation field to the $\mathrm{CC}$ segmentation on the template to segment the $\mathrm{CC}$ on the extracted slice 17 . The area of the deformed $\mathrm{CC}$ on the template is taken to be the area of $\mathrm{CC}$ on the extracted slice. We use deformable registration using the discrete optimization (DROP) method [18] because it is initialization-free, does not require gradient calculations, and the Markov random field energy it adopts is optimized quickly using an efficient primal dual approach [19]. We use the sum of squared intensity differences as the similarity metric because the template and target come from the same scan.

\subsection{Area Optimization}

We minimize $A_{c c}$ with respect to $P_{\text {ext }}$. During each iteration of the optimizer, $\frac{\partial A_{c c}}{\partial T_{z}}, \frac{\partial A_{c c}}{\partial R_{x}}$ and $\frac{\partial A_{c c}}{\partial R_{y}}$ are calculated using central differencing. A sequential quadratic programming method is employed for optimization. The optimizer updates an estimate of the Hessian of the Lagrangian at each iteration using the BFGS formula 20, 21. We set the lower and upper search bounds empirically at $-2.0^{\circ}$ and $2.0^{\circ}$ for $R_{x}$ and $R_{y}$, and at $-2.0 \mathrm{~mm}$ and $2.0 \mathrm{~mm}$ for $T_{z}$. The 
bounds are in place largely for computational tractability, but they also ensure that the optimizer does not converge to a degenerate case in which a plane does not intersect with the CC bridge and has therefore zero CC area.

\subsection{Multi-level Optimization}

In addition to initializing with the MSP, to further decrease the possibility of converging to a local minimum of the MCCAP, we use a multi-level optimization scheme, where, in each step, the level of image smoothness is decreased and the optimization algorithm is initialized at the MCCAP parameters from the previous step. Smoothing is realized via 3D isotropic Gaussian filters with standard deviations: $10,5,3,2,1.8,1.6,1.4, \ldots, 0.6,0.4$, and $0.2 \mathrm{~mm}$ (set empirically based on $3 \mathrm{D}$ visualizations of the variations in $\mathrm{CC}$ area over the entire search space, computed using brute force search in several sample MRI volumes). The size of each filter in each dimension is five times the standard deviation.

\section{Results}

We performed three experiments. In the first two experiments (Sec. 3.1 and Sec. 3.2), we used 3D T1 MRI brain scans of 20 normals from the internet brain segmentation repository (IBSR)(http://www.cma.mgh.harvard.edu/ibsr/). Each volume consists of 60 to 65 coronal slices, each of dimensions $256 \times 256$, and was positionally normalized using the midpoints of the decussations of the anterior and posterior commisures and the midsagittal plane at the level of posterior commisure as points of reference for rigid transformation [22. The repositioned scans were then re-sliced into $3 \mathrm{~mm}$ thick coronal slices with $1 \times 1 \mathrm{~mm}$ pixels. In the third experiment (Sec. 3.3), we used 3D T1 MRI axial brain volumes of 10 secondary progressive MS patients and 5 normals. Each dataset dimension is $256 \times 256 \times 160$, with voxel size $1.17 \times 1.17 \times 1 \mathrm{~mm}$. The MSP was extracted by an MRI technologist trained on the Philips Achieva 3.0 Tesla scanner by initiating a 3-plane localizer in the coronal, axial, and sagittal planes, using the interhemispheric fissure as the internal landmark, and correcting for ear to shoulder tilt on the coronal plane and right to left rotation on the axial plane. We use an MSP obtained by careful manual placement because it gives us greater confidence in accuracy than automatic MSP methods that would still have to be visually inspected.

\subsection{Achievement of Global Minima}

We show that the MCCAP area found by the proposed optimization method is generally very close to the ground truth. We performed a brute force $(\mathrm{BF})$ search in the space of $T_{z}, R_{x}$ and $R_{y}$, with search resolution $0.1 \mathrm{~mm}, 0.1^{\circ}$, and $0.1^{\circ}$, respectively, and calculated the $\mathrm{CC}$ area on each slice to find the minimal $\mathrm{CC}$ area, up to the resolution of the search parameters. The upper and lower limits on $P_{\text {ext }}$ were set as in Sec. 2.4. We compared the CC areas on the MCCAP using our 


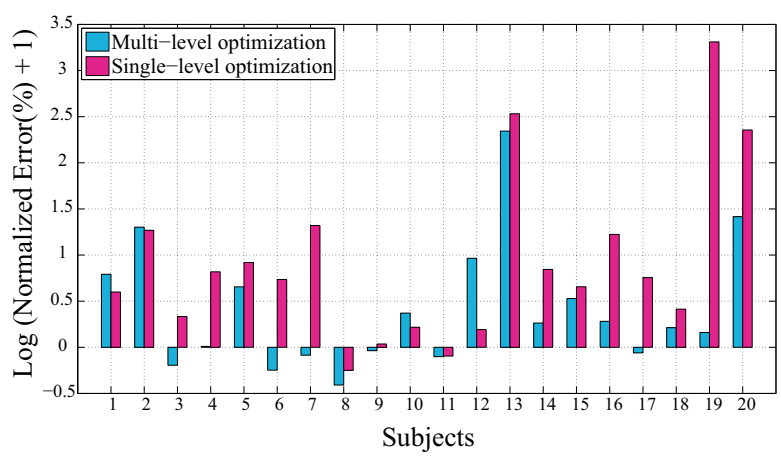

Fig. 2. Normalized error (\%) in MCCAP areas (shown in logarithmic scale) is calculated as the difference between the MCCAP area computed by either optimization method and the minimum CC area computed by brute force, normalized by the minimum brute force area. The results from the multi-level optimization method is in general closer to the ground truth (global optimum). $\log (x+1)$ plot is used to take care of zero and negative values.

optimization algorithm with single- (Sec. 2.4) and multi-level optimization (Sec. 2.5) with BF results. With BF considered as ground truth, the error is computed as: Normalized Error $(\%)=\left(\right.$ Area $_{\mathrm{MCCAP}}-$ Area $\left._{\mathrm{BF}}\right) / \operatorname{Area}_{\mathrm{BF}} \times 100 \%$ (Fig. 2). In all cases except one, the minimum area from multi-level smoothing optimization is within $3.12 \%$ of the corresponding brute force result. In 7 cases, the minimum area from multi-level method is even smaller than brute force result due to the limited resolution of brute force search. The multi-level method produced a lower MCCAP area in comparison to the single-level in $85 \%$ of the cases (17 out of 20 subjects) by up to $26 \%$. In the remaining 3 cases, the $\mathrm{CC}$ areas on the MCCAP found using the single-level optimization are slightly smaller (within 1.4\%) than the multi-level counterpart, which may be due to the parameters of the multilevel smoothing resulting in over fitting of the optimizer at lower resolutions. This supports the assertion that the multi-level method yields results closer to the ground truth.

To investigate the uniqueness of the MCCAP, we compared the MCCAP parameters from the multi-level and brute force methods, and found that the parameters of the extracted planes can be substantially different (differences in $T_{z}=0.5 \pm 1.2 \mathrm{~mm}, R_{x}=0.51 \pm 0.66^{\circ}$, and $R_{y}=0.65 \pm 0.82^{\circ}$ ), even when their computed minimal areas are very close. This supports the intuition that the MCCAP is not necessarily unique for a given brain MRI volume.

\subsection{Quantitative Results for Method Robustness}

To evaluate the robustness of our method, we compared the CC areas on the MCCAP in all 20 subjects for different initializations with respect to a default initialization of $T_{z}=R_{x}=R_{y}=0$. The different initializations simulate minor variations in head position or slice angle, assuming some effort has been made 


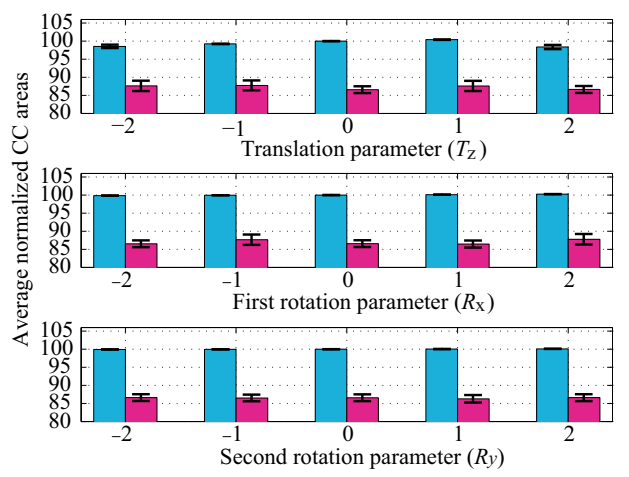

Fig. 3. Averaged normalized MCCAP areas for different initializations (while varying each parameter, the other two are set to zero) are shown in pink bars, while blue bars show the averaged normalized $\mathrm{CC}$ area on the initial slice. Normalization is done to the $\mathrm{CC}$ area on the slice with default initialization. Convergence of our method to almost the same normalized MCCAP area for different initializations demonstrates the robustness of the proposed method.

to center the volume. Fig. 3 shows that our method is robust to a wide range of initializations and is likely to converge very closely to a global minimum. The variance in MCCAP areas in all initializations is less than 1.4\%. Also note that comparing the mean error between the MCCAP and brute force (from section (3.1) which is $1.08 \%$ with the $9-10 \%$ reduction in Fig. 3 shows that we recover approximately $90 \%$ of area difference between MSP and brute force.

\subsection{MCCAP vs. MSP in Multiple Sclerosis Patients}

We compared the discriminatory value of the CC areas calculated using an MSP vs. the MCCAP in distinguishing between multiple sclerosis (MS) patients and healthy controls. The means and standard deviations of the $\mathrm{CC}$ areas computed using the MSP and two MCCAP optimization methods are shown in Fig. 4, The multi-level smoothing optimization seems to be the best method at distinguishing the two groups. Note the larger separation of areas between the MS and normal groups using MCCAP multi-level optimization, as compared to the MSP. The area reduction for the normals $(\sim 12 \%)$ is much lower than for the patients $(\sim 50 \%)$, which we attribute to the true $3 \mathrm{D}$ shape differences between the groups. We quantified the advantage by computing the standardized mean differences between the normal and MS groups for MSP and MCCAP values using $(\overline{\text { Normal }}-\overline{\mathrm{MS}}) / \sqrt{\sigma_{\mathrm{Normal}^{2}} / n_{\text {Normal }}+\sigma_{\mathrm{MS}^{2}} / n_{\mathrm{MS}}}$, where $\sigma$ is the

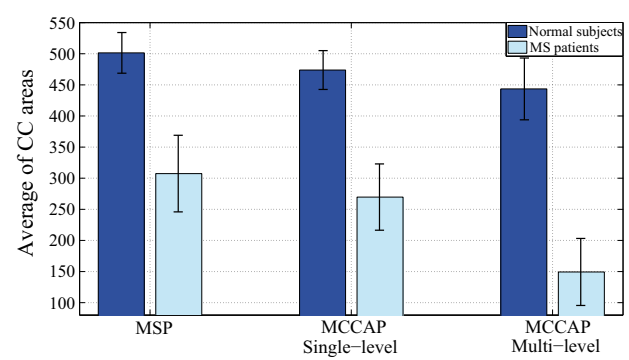

Fig. 4. Average of CC areas using different methods in MS patients and normal subjects. The larger difference in MCCAP areas found by multi-level smoothing optimization method shows that the two groups can be better distinguished than when MSP or singlelevel MCCAP areas are used. 
standard deviation of each group and $n$ is the sample size. If a method yields a bigger standardized mean difference, this suggests that it is better at distinguishing the two groups. The standardized mean difference for multi-level optimization is 10.51 which, compared to 7.97 for the MSP, is a $24 \%$ increase. Further, the p-values, associated with rejecting the hypothesis that the two populations come from the same distribution, show statistically significant differences between the normal and the MS subjects for all of the tested methods: $p_{\mathrm{MSP}}=1.94 \mathrm{e}-5, p_{\mathrm{MCCAP}-\text { single }}=2.82 \mathrm{e}-6, p_{\mathrm{MCCAP}-\text { multi }}=1.4 \mathrm{e}-7$. These initial results are supportive of our hypothesis that the interhemispheric communication bottleneck is an important bio-marker for MS, and that the MCCAP is better motivated than MSP to measure this bottleneck.

\section{Conclusion}

The plane with minimum CC area is proposed for the first time as the plane for studying CC atrophy. This choice is based on the hypothesis that the MCCAP is the cross section that is most representative of the bottleneck of interhemispheric transmission. We have developed an accurate and largely automatic method that employs an optimization process for extraction of the MCCAP along with the simultaneous segmentation of the embedded CC. We have shown that our optimizer converges closely to the global optimum area and is robust with respect to initialization. Preliminary results demonstrate better class separation between MS patients and normal subjects when the areas from the MCCAP are compared to those from an MSP. Future work includes longitudinal studies with larger data sets and more rigorous statistical tests (e.g. not necessarily assuming a normal distribution) to assess the usefulness of the MCCAP in monitoring disease progression and response to therapy.

\section{Acknowledgements}

Ghassan Hamarneh, Neda Changizi, and Omer Ishaq were partially funded by the Natural Sciences and Engineering Research Council of Canada. Advice on statistical methods was provided by Yinshan Zhao from Faculty of Medicine (Neurology), University of British Columbia.

\section{References}

1. Aboitiz, F., Scheibel, A.B., Fisher, R.S., Zaidel, E.: Fiber composition of the human corpus callosum. Brain Res. 598(1-2), 143-153 (1992)

2. Simon, J.H., et al.: A longitudinal study of brain atrophy in relapsing multiple sclerosis. Neurology 53(1), 139-148 (1999)

3. Woodruff, P.W., McManus, I.C., David, A.S.: Meta-analysis of corpus callosum size in schizophrenia. J. Neurol. Neurosurg. Psychiatry 58(4), 457-461 (1995)

4. Hardan, A.Y., Minshew, N.J., Keshavan, M.S.: Corpus callosum size in autism. Neurology 55(9), 1033-1036 (2000)

5. Smith, S., Jenkinson, M.: Accurate robust symmetry estimation. In: Taylor, C., Colchester, A. (eds.) MICCAI 1999. LNCS, vol. 1679, pp. 308-317. Springer, Heidelberg (1999) 
6. Ardekani, B.A., Kershaw, J., Braun, M., Kanuo, I.: Automatic detection of the mid-sagittal plane in 3-D brain images. IEEE TMI 16(6), 947-952 (1997)

7. Liu, Y., Collins, R., Rothfus, W.: Robust midsagittal plane extraction from normal and pathological 3D neuroradiology images. IEEE TMI 20(3), 175-192 (2001)

8. Brummer, M.E.: Hough transform detection of the longitudinal fissure in tomographic head images. IEEE TMI 10(1), 74-81 (1991)

9. Marais, P., Guillemaud, R., Sakuma, M., Zisserman, A., Brady, M.: Visualising cerebral asymmetry. In: Höhne, K.H., Kikinis, R. (eds.) VBC 1996. LNCS, vol. 1131, pp. 411-416. Springer, Heidelberg (1996)

10. Anbazhagan, P., Carass, A., Bazin, P.L., Prince, J.L.: Automatic estimation of midsagittal plane and AC-PC alignment on nonrigid registration. In: IEEE ISBI, pp. 828-831 (2006)

11. Ishaq, O., Hamarneh, G., Tam, R., Traboulsee, A.: Effects of Mid Sagittal Plane Perturbation and Image Interpolation on Corpus Callosum Area Calculation. In: IEEE ISSPIT, pp. 197-202 (2006)

12. Skoglund, K.V., Stegmann, M.B., Ryberg, C., Ólafsdóttir, H., Rostrup, E.: Estimation and Perturbation of the Mid-Sagittal Plane and its Effects on Corpus Callosum Morphometry. In: International Society of Magnetic Resonanc in Medicine (2005)

13. Barkhof, F.J., Elton, M., Lindeboom, J., Tas, M.W., Schmidt, W.F., Hommes, O.R., Polman, C.H., Kok, A., Valk, J.: Functional correlates of callosal atrophy in relapsing-remitting multiple sclerosis patients. A preliminary MRI study. J. Neurol. 245(3), 153-158 (1998)

14. Bonzano, L., Tacchino, A., Roccatagliata, L., Abbruzzese, G., Luigi Mancardi, G., Bove, M.: Callosal contributions to simultaneous bimanual finger movements. J. Neurosci. 28(12), 3227-3233 (2008)

15. Pozzilli, C., Bastianello, S., Padovani, A., Passafiume, D., Millefiorini, E., Bozzao, L., Fieschi, C.: Anterior corpus callosum atrophy and verbal fluency in multiple sclerosis. Cortex 27(3), 441-445 (1991)

16. Pelletier, J., Suchet, L., Witjas, T., Habib, M., Guttmann, C.R., Salamon, G., Lyon-Caen, O., Chérif, A.A.: A longitudinal study of callosal atrophy and interhemispheric dysfunction in relapsing-remitting multiple sclerosis. Arch. Neurol. 58(1), 105-111 (2001)

17. Collins, D.L., Holmes, C.J., Peters, T.M., Evans, A.C.: Automatic 3-D model-based neuroanatomical segmentation. Human Brain Mapping 3(3), 190-208 (1995)

18. Glocker, B., Komodakis, N., Tziritas, G., Navab, N., Paragios, N.: Dense Image Registration through MRFs and Efficient Linear Programming. Med. Image Anal. 12(6), 731-741 (2008)

19. Komodakis, N., Tziritas, G., Paragios, N.: Fast, Approximately Optimal Solutions for Single and Dynamic MRFs. In: IEEE CVPR, pp. 1-8 (2007)

20. Fletcher, R., Powell, M.J.D.: A Rapidly Convergent Descent Method for Minimization. Computer Journal 6, 163-168 (1963)

21. Goldfarb, D.: A Family of Variable Metric Updates Derived by Variational Means. Mathematics of Computing 24, 23-26 (1970)

22. Talairach, J., Tournoux, P.: Co-planar stereotaxic atlas of the human brain. Thieme, New York (1988) 